\title{
Publishers: Save Authors' Time
}

\author{
Khaled Moustafa ${ }^{1}$
}

Received: 12 January 2017/ Accepted: 17 January 2017

(C) Springer Science+Business Media Dordrecht 2017

\begin{abstract}
Scientific journals ask authors to put their manuscripts, at the submission stage, sometimes in a complex style and a specific pagination format that are time consuming while it is unclear yet that the submitted manuscripts will be accepted. In the case of rejections, authors need to submit to another journal most likely with a different style and formatting that require additional work and time. To save authors' time, publishers should allow authors to submit their manuscripts in any format and to comply with the style required by the targeted journal only in revised versions, but not at the submission step when the manuscripts are not yet approved for publication.
\end{abstract}

Keywords Publishing scientific paper - Manuscript submission - Manuscript writing - Manuscript rejection - Manuscript acceptance - Publication ethics ·

Publication bias · Publishing ethics · Instruction to authors · Instructions for authors

Scientific journals have different citation styles and page setup requirements. Each style has its own pros and cons, though some styles are more or less complex than others. No one style, however, seems to make an agreement accepted by all the journals and all the authors as each style has its proponents and opponents with valid arguments for each style. As such, and prior to submission, journals require authors to put their manuscripts in accordance with their preferred styles, which are sometimes overwhelming and time consuming. If the submitted manuscript is accepted, anyway, this will be somewhat justified by the reward of manuscript acceptance. However, in case of repetitive rejection, this will make authors waste time in unnecessary formatting styles particularly when authors are compelled to submit their rejected manuscripts to other journals with different styles and formats.

Khaled Moustafa

khaled.moustafa@gmail.com

1 Conservatoire National des Arts et Métiers, Paris, France 
To save authors' time and avoid authors' frustration in long and unnecessary formatting setups, journals may need to differentiate between revised manuscripts and new submissions. That is, authors should be able to submit their manuscripts in any format as long as the format is consistent and readable. Then, only when the manuscript is accepted, the authors could be asked to follow the style required by the targeted journal. Such a solution is easily and immediately applicable by all journals, whatever the journal's opted style. It does not involve any additional costs or efforts for journals or publishers. All that journals need to do is simply to add a short sentence in their instructions for authors indicating that it is not necessary to format manuscripts according to any special style. However, if the submitted manuscript is accepted, the authors will be asked to apply the citation style of the targeted journal in a revised version. Doing so, the submission process will be simplified (Moustafa 2015) and authors will avoid time wasting and frustration.

\section{Reference}

Moustafa, K. (2015). Blind manuscript submission to reduce rejection bias? Science and Engineering Ethics, 21(2), 535-539. 